\title{
Silk evolution untangled
}

The complex proteins that make up spider silks, which are extruded threads that have evolved to work in the dry state, are easily studied and provide us with windows on to protein folding, synthetic biology and nanomaterials. Silks have diversified through selection over millennia to produce thousands of types, and are superbly fit for their purposes.

Alas, silks form only a small part of Leslie Brunetta and Catherine Craig's book. Spider Silks focuses mainly on spider ecology and web evolution. It is therefore appropriate for the engineer, materials scientist or chemist who is attracted to silk research but needs a grounding in biological processes. Those seeking detailed insight into the silks themselves will be disappointed. The authors science writer Brunetta and biologist Craig — briefly discuss the variety of silks and their molecular structures and functions. But they quickly bury that information in explana-

the water to roam the land some 400 million years ago. Early spiders used thin films of flimsy silk filaments to build shelters or div-

Spider Silk: Evolution and $\mathbf{4 0 0}$ Million Years Spinning, Waiting, Snagging, and Mating by Leslie Brunetta and Catherine L. Craig Yale University Press: 2010. 248 pp. \$30, £20

and more functional silks.

With its spider focus, the book does not discuss silks made by other taxa, such as moths and bees. Silk has evolved several times independently, yet it shares many traits - as well as diversity - between different organisms. Spider silks, which stem from one ancestor, show the greatest variation within one taxon in both chemical composition and production methods. Comparison of spider and other silks tells us about the molecular processes of biopolymer evolution - and of proteins in particular.

Silks consist largely of fibrous proteins. Their complex, long amino-acid sequences hold the keys to silk's remarkable properties: tions of mechanisms of inheritance and arachnid ecology.

The book's limited focus is a missed opportunity because the evolution of web materials and structures cannot be disentangled. Although webs are the obvious signature of a spider's ecology, silks are the key to the success of this diverse taxon. By allowing the efficient construction of a variety of lightweight nets, silks have enabled spiders to inhabit numerous ecological niches across a competitive landscape that also features huge insect diversity.

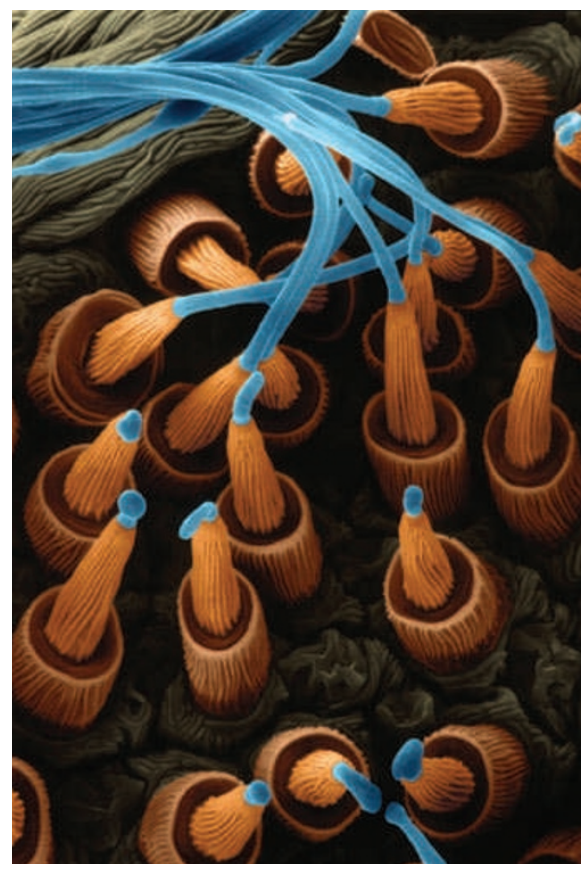

Silk secretion: the key to spider success.
The earthbound snares of early spiders may have caused some insects to evolve wings and take to the air to escape them. The book introduces both fossil spiders and living fossils, the abdominal segmentation and silk spinning spools of which resemble those of ancestors that left its ability to be stored for months, its rapid transformation from liquid to solid and the strength and flexibility of the finished fibre that often outperforms man-made materials. These qualities of silk, combined with biodegradability and biocompatibility, make it of interest to both biologists and clinicians - in tissue engineering and medical implants, for example. Although it is a one-sided account, Spider Silks shows that, with so many applications, silks have as bright a future as commercial mulberry silks had a past.

Fritz Vollrath is a senior researcher and visiting professor at the Department of Zoology, University of Oxford, South Parks Road, Oxford OX13PS, UK, and head of the Oxford Silk Group.

e-mail: fritz.vollrath@zoo.ox.ac.uk

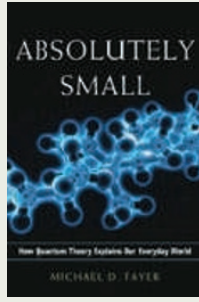

In Absolutely Small (McGraw Hill, 2010), chemist Michael Fayer explains the weird microscopic behaviour of matter. Favouring everyday

examples over formulae, he makes quantum mechanics palatable, from wave-particle duality to the uncertainty principle. Beer, soap and fat are the ingredients he chooses to explain how chemical bonds work. With summaries of the chemistry of greenhouse gases, hydrocarbons and the electrical properties of materials, Fayer's book provides a useful overview.

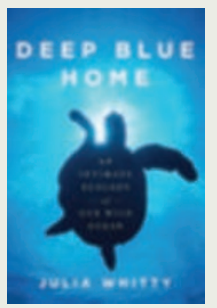

Former scientist and film-maker Julia Whitty relates a personal view of ocean ecology in Deep Blue Home (Houghton Mifflin Harcourt, 2010).

Travelling from the Sea of Cortez to Newfoundland and the Galapagos Islands, she describes encounters with whales and turtles, and the work of the scientists who are trying to protect and understand them. While explaining how the world's oceans are unified by deep currents, she also reveals their effect on Earth's climate.

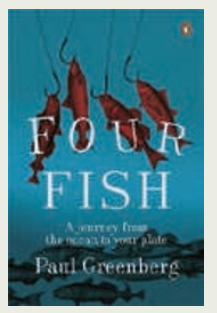

Dwindling fish stocks form the focus of Paul Greenberg's book. Four Fish (Allen Lane, 2010) looks at the quartet of species that we eat most - salmon, sea bass, cod and tuna. The writer travelled from Norway to the South Pacific to see how these animals reach our plates and how they are threatened by overfishing. Posing pragmatic questions such as how to find sustainable seafood on a supermarket shelf, he weaves together the biology of sea fish, fisheries and conservation issues. He ends with a proposal for choosing the species that we can farm without disrupting wild ecosystems.

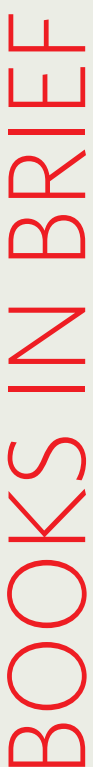

